Classification

Physics Abstracts

$78.20-81.15$

\title{
Laterally graded division parameter layered synthetic microstructures
}

\author{
C. Guichet $\left({ }^{1}\right)$, R. Rivoira $\left({ }^{1}\right)$, G. Rasigni $\left({ }^{1}\right)$ and A. Charai $\left({ }^{2}\right)$
}

( ${ }^{1}$ ) Département de Physique des Interactions Photons-Matière Case EC1, Faculté des Sciences de St Jérôme, 13397 Marseille Cedex 20, France

( $\left.{ }^{2}\right)$ Laboratcire de Métallurgie U.R.. 443 C.N.R.S. Case 511, Faculté des Sciences de St Jérôme, 13397 Marseille Cedex 20, France

(Received May 25; accepted July 15, 1994)

\begin{abstract}
Résumé. - Des microstructures synthétiques en couches minces à variation latérale de $\gamma$ (oú $\gamma$ est le rapport entre l'épaisseur de matériau de haut indice et le paramètre de la multicouche) ont été fabriquées par pulvérisation. Elles ont été caractérisées par réflectivité spéculaire $\mathrm{X}$ avec un diffractomètre fonctionnement sous rayonnement primaire monochromatique $\mathrm{Cu} \mathrm{K}_{\alpha}$. Les résultats sont en bon accord avec ceux prévus par la théorie dynamique de la diffraction $\mathrm{X}$, ainsi qu'avec des simulations sur ordinateur et des observations en MET. Nous montrons ainsi qu'il est possible de réaliser des structures dont l'intérêt est de disposer sur un échantillon de valeurs du paramètre $\gamma$ correspondant à des applications diverses.
\end{abstract}

\begin{abstract}
A laterally graded $\gamma$ layered synthetic microstructure (LSM), (where the division parameter $\gamma$ is the ratio between the thickness of the high refractive index material and the LSM's period), has been manufactured by means of a sputtering technique. This special LSM has been characterized by specular reflectivity $\mathrm{X}$-ray measurements using a diffractometer equipped with an incident beam monochromator and $\mathrm{Cu}-\mathrm{K}_{\alpha}(1.5418 \AA)$ radiation. It exhibits the properties expected by the dynamical theory of X-ray diffraction. The results are shown to be in good agreement with computer simulations and transmission electron microscopy (TEM) observations. The object of this paper is to prove the feasibility of such a structure whose the interest is the selection on one sample of the division parameter $\gamma$ desired for different applications. We give a calibration curve of the sample by means of an experimental law of laterally $\gamma$ variation.
\end{abstract}

\section{Introduction.}

During the last decade remarkable progress in thin films technology has made possible the designing of layered synthetic microstructures (LSMs) that act as efficient Bragg diffractors in the $\mathrm{X}$-rays and extreme ultraviolet spectral ranges [1-4]. These LSMs, which consist of alternating layers of high and low refractive index are analogous to multilayer interference filters for the vis- 
ible spectral region. By adjusting the refractive indices and thicknesses of the component layers the diffracting properties may be tailored to specific applications: X-ray telescope, X-ray laser, $\mathrm{X}$-1 ay microscopy, X-ray lithography etc [5].

Some laboratories have shown great skill in manufacturing very high quality LSMs with essentially atomically smooth interfaces between the constituant layers. A large variety of special structures have been achieved: for instance Fabry-Perot etalon for X-rays [6,7], laterally graded period LSM [8,9], multilayer laminar grating [10], etc..., only limited by the constraints of the technology now available. In this paper we consider a structure which, to our knowledge, has not been designed yet, namely a laterally graded $\gamma$ LSM, where $\gamma$ is the ratio $d_{\mathrm{H}} /\left(d_{\mathrm{H}}+d_{\mathrm{L}}\right), d_{\mathrm{H}}$ and $d_{\mathrm{L}}$ being thicknesses of the high and low refractive index layer, respectively. The interest in manufacturing and characterizing such a structure is twofold. First, the dynamical theory of X-ray diffraction shows that for particular $\gamma$ values, only some peculiar Bragg reflections are allowed. Such a prediction needs to be checked in order to determine to what extent the dynamical theory may be used to explain LSM's properties. Second, this structure might be found useful in X-ray spectroscopy [11]. Indeed, if an experimental set-up has to work with the first Bragg order for the main wavelength $\lambda_{0}$ of the X-ray source, higher Bragg orders of $\lambda_{0}$-harmonics could disturb specular reflections by diffraction in the same direction. So, it seems to be appropriate to have a structure which can be adapted by the choice of the parameter $\gamma$ for the suppression of these undesirable reflections. Moreover, in several applications, the second order (or higher orders) have to be used because of constraints of incidence angle. By the choice of the parameter $\gamma$, it is possible to increase the efficiency in this order by suppressing higher orders [12].

\section{Theoretical model for LSMs.}

Two theoretical approaches may be used to describe the reflection of X-rays from a natural crystal or a LSM [13]:

- the kinematical theory (KT)

- the dynamical theory (DT)

The KT neglects the details of the wave interactions when DT takes all wave interactions into account. The DT is usually used in the study of the X-ray diffraction from large perfect crystals. Given that LSMs may be considered as perfect from crystallographic point of view, it seems more appropriate to use the DT for studying their diffracting properties. Moreover the neglect of absorption in layer materials makes it possible to have a simplified theory for deducing some expressions in closed form [14].

The peak reflectivity $R_{m}^{(p)}$ for the $m$ th Bragg reflection is defined as the maximum value of $I(\theta) / I_{0}$, where $I_{0}$ and $I(\theta)$ are the intensities of the incident and reflected waves respectively. The dynamical theory provides $[13,14]$

$$
R_{m}^{(p)}=\mathrm{th}^{2} B
$$

where $B=\left(2 N d P\left(\theta_{m}\right)\left|F\left(\theta_{m}\right)\right|\right) / m, \theta_{m}$ is the Bragg angle, $N$ is the number of layer pairs, $F\left(\theta_{m}\right)$ is the structure factor, and

$$
\begin{cases}P\left(\theta_{m}\right)=1 & \text { for the } \sigma \text { - polarization } \\ P\left(\theta_{m}\right)=\cos 2 \theta_{m} & \text { for the } \pi \text { - polarization } \\ P\left(\theta_{m}\right)=\left(1+\cos 2 \theta_{m}\right) / 2 & \text { for unpolarized radiation. }\end{cases}
$$

In the case of a LSM made of alternating homogeneous layers of a high refractive indice material $\mathrm{H}$ and a low refractive indice material $\mathrm{L}, F\left(\theta_{m}\right)$ may be written as:

$$
F\left(\theta_{m}\right)=(d / m \pi) \sin \left[\left(r r \pi d_{\mathrm{H}}\right) / d\right]\left(\phi_{\mathrm{H}}-\phi_{\mathrm{L}}\right)
$$


where $\phi_{\mathrm{H}}$ and $\phi_{\mathrm{L}}$ are the scattering amplitude densities of materials $\mathrm{H}$ and L, respectively.

Usually the ratio $d_{\mathrm{H}} / d$ is called $\gamma$. Hence the structure factor depends on $\gamma$ according to

$$
F\left(\theta_{m}\right)=(d / m \pi) \sin (m \pi \gamma)\left(\phi_{\mathrm{H}}-\phi_{\mathrm{L}}\right)
$$

This equation shows that an extinction of the $m$ th Bragg order can be expected for discrete values of $\gamma$ such that

$$
\gamma=k / m, \quad 1 \leq k \leq m-1
$$

Especially only the odd order $(m=1,3,5 \ldots)$ reflections are permitted for $\gamma=0.5$.

\section{Multilayer design.}

Because of its appealing properties [15] - mainly high stability and high scattering power per layer pair - the tungsten/carbon (W/C) system has been chosen to manufacture our samples.

These samples are prepared using a triode DC sputtering technique under low argon pressure $\left(2 \times 10^{-3} \mathrm{Torr}\right)$. The temperature of the targets is regulated. The substrates are (111) silicon wafers of 6 inches $(15 \mathrm{~cm}$ ) with a rms (root mean square) roughness height estimated to be about $0.5 \mathrm{~nm}$. The deposition rates are about $0.2 \mathrm{~nm} / \mathrm{s}$ for $\mathrm{W}$ and $0.03 \mathrm{~nm} / \mathrm{s}$ for C. Figure 1 a shows the experimental set-up.

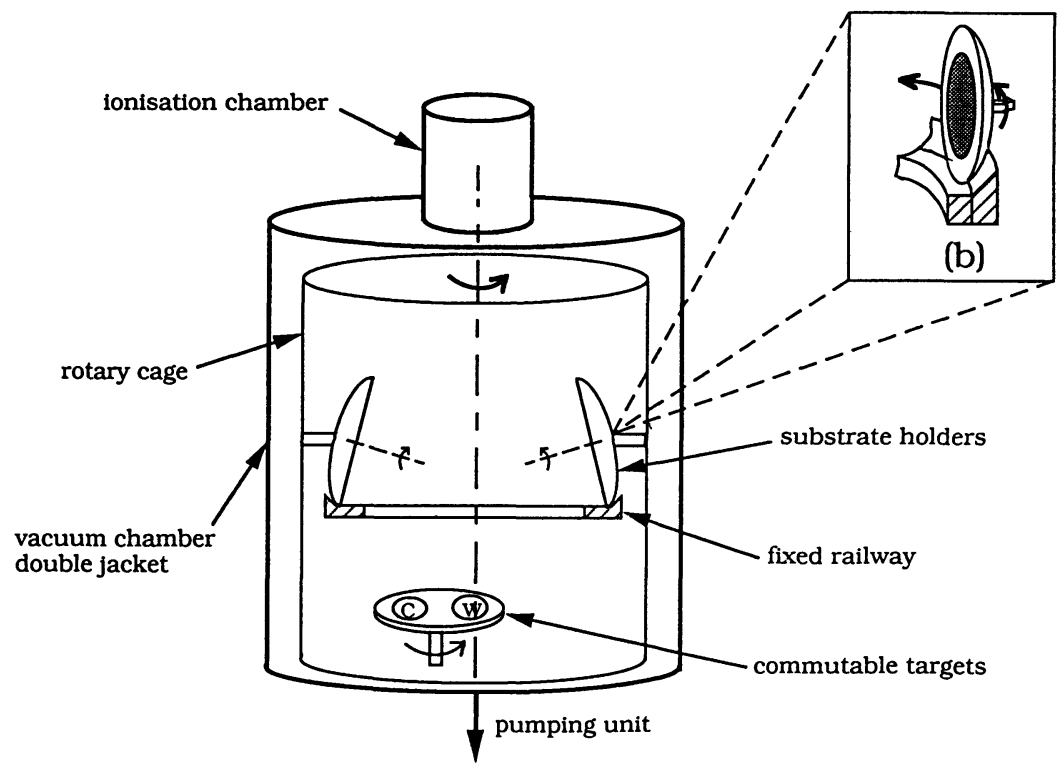

(a)

Fig. 1. - a) Experimental set-up and b) planetary drive on runner ring.

In order to understand how a laterally graded $\gamma$ LSM can be manufactured we have first to describe the method leading to an homogeneous LSM. The process is as follows. 
The Si substrates are put on a planetary drive which is held by a roller ring. This roller ring, which is uniformely moved about a vertical axis by means of an electrical engine, makes the planetary drive to turn about its axis of rotation by sliding friction on a fixed railway (Fig. 1b). So during the vaporisation the substrates undergo both a rotary motion about the axis of rotation of the planetary drive. This makes it possible to manufacture homogeneous samples with an excellent film uniformity and optimum step coverage, as it has been shown previously [16] and despite the fact there is a steep deposition angle between the targets and the substrates (approximatively $\left.40^{\circ}\right)$.

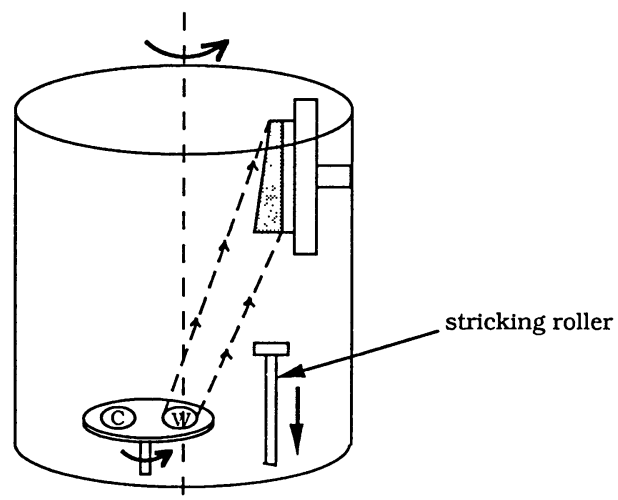

(a)

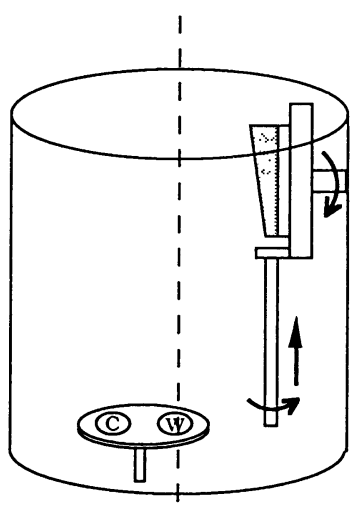

(b)

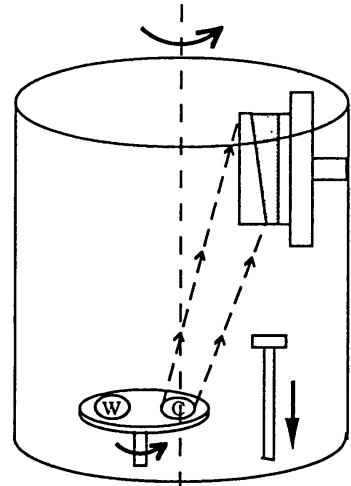

(c)

Fig. 2. - Manufacture of a laterally graded $\gamma$ LSM. a) Deposition of a laterally graded thickness film for the first material (W). The planetary drive is deconnected from the runner ring; b) Rotation of $180^{\circ}$ of the planetary drive by means of a stricking roller; c) Deposition of the second material (C) like the first one.

In order to obtain a laterally graded $\gamma$ LSM we must carry out the experiment in three steps (Fig. 2). First the planetary drive is disconnected from the fixed railway. So the substrates are only driven in a rotation motion about a vertical axis. This allows us to obtain a film with a laterally graded thickness for the first material (W) (Fig. 2a). The process is stopped. The planetary drive is rotated by $180^{\circ}$ about its rotation axis (Fig. 2b) by means of a stricking roller and the second 
material (C) is deposited like the first one, namely with the substrate undergoing a rotary motion about the vertical axis only (Fig. 2c). The process is repeated until all the layers are obtained. Let us remark that deposition occurs at a steep angle but several microscopy studies on various LSMs have shown that this does not influence roughness.

\section{X-ray diffraction characterization.}

Three samples have been manufactured by means of the above described process. We deal with a W/C LSM made of 31 bilayers, approximately $3 \mathrm{~nm}$ period, whose theoretical profile is shown in figure 3. This sample has been characterized by specular reflectivity using a diffractometer equipped with an incident beam monochromator and $\mathrm{Cu}-\mathrm{K}_{\alpha}(1.5418 \AA)$ radiation. The measurements have been performed at regularly spaced points $(\mathrm{cm}$ per $\mathrm{cm})$ along the $x$ direction of the sample (Fig. 3). The period $d$ of the sample and the relative intensities of the $2^{\text {nd }}$ and $3^{\text {rd }}$ order Bragg peaks with regard to the $1^{\text {st }}$ one can be retrieved from the diffraction spectrum.

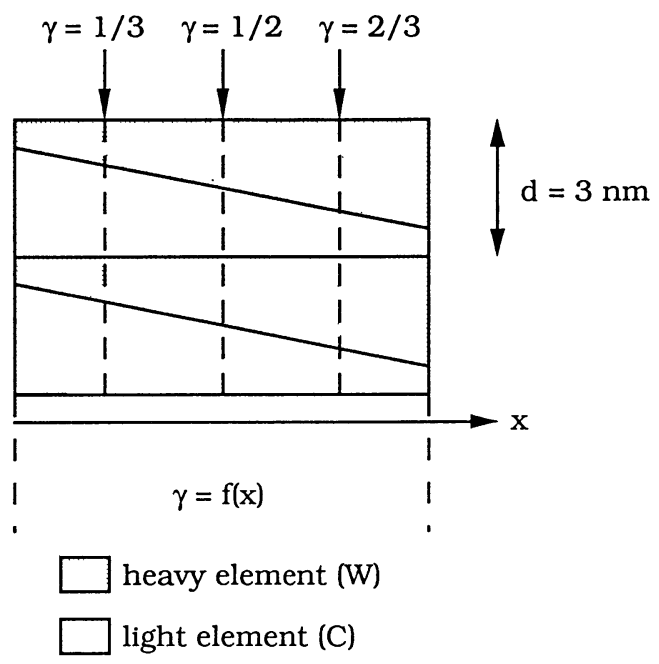

Fig. 3. - Theoretical profile for a laterally graded $\gamma$ LSM.

First we have to verify that the sample period $d$ is approximately constant along the $x$ direction and close to the expected value $3 \mathrm{~nm}$. The calculation of $d$ from Bragg angles has been carried out by means of the corrected Bragg's law which takes dispersion into account, namely [17]

$$
2 d \sin \theta_{n}\left[1-2 \bar{\delta} / \sin ^{2} \theta_{n}\right]^{1 / 2}=n \lambda,
$$

where $n$ is the diffraction order, $\bar{\delta}$ is the mean decrement defined as:

$$
\bar{\delta}=\gamma \delta_{\mathrm{H}}+(1-\gamma) \delta_{\mathrm{L}},
$$

where $\delta_{\mathrm{H}}$ and $\delta_{\mathrm{L}}$ are the high and low refractive index material decrements respectively. It ensues 
that

$$
\sin ^{2} \theta_{n}=\left(\lambda^{2} / 4 d^{2}\right) n^{2}+2 \bar{\delta}
$$

There is a linear relation between $\sin ^{2} \theta_{n}$ and $n^{2}$. If we take into account the first three orders simultaneously, it is possible from a least square fit of the data to deduce the gradient $\lambda^{2} / 4 d^{2}$, and later on $d$. The results are reported in table I. It can be seen that the period is approximately constant along the $x$ direction (within the accuracy range of measurements) and close to the expected value $3 \mathrm{~nm}$.

Table I. - Experimental period vs $x$ deduced from the diffraction spectra computed by X-ray characterization.

\begin{tabular}{cc}
\hline $\mathrm{x}(\mathrm{cm})$ & Period $(\mathrm{nm})$ \\
\hline 1 & 3.3 \\
2 & 3.1 \\
3 & 3.2 \\
4 & 3.0 \\
5 & 3.1 \\
6 & 3.1 \\
7 & 3.0 \\
8 & 3.0 \\
10 & 2.9
\end{tabular}

Figure 4 shows, as a function of $x$, the relative intensities of the $2^{\text {nd }}$ and the $3^{\text {rd }}$ order Bragg peaks with respect to the first one. For convenience the two curves are represented on the same figure. It is clear from this figure that the experimental behavior of the sample is in good agreement with predictions based on the dynamical theory. In particular the $3^{\text {rd }}$ order reflectivity is at a peak when the $2^{\text {nd }}$ order is practically zero. According to the DT such a behavior means that the experimental values of $\gamma$ belong to a range nearly centered on $\gamma=0.5$, which is connected with an extinction for the second order reflectivity. It must be emphasized that the $3^{\text {rd }}$ order reflectivity displays a high ratio of noise to signal because of the very low intensity measurements related to this order.

At this stage of our study we decided to assign the value $\gamma=0.5$ to the point located at $x=5$ $\mathrm{cm}$. This value is corroborated by transmission electron microscopy (see later on). For practical applications it is important to determine the experimental law $\gamma=f(x)$ which may be used as the calibration curve. Such a determination requires the knowledge of the interface slope between the $\mathrm{C}$ and $\mathrm{W}$ layers. Actually this slope may be deduced from the geometry of the experimental set-up. Preliminary experiments [9] related to a LSM with a laterally graded period enable us to determine this slope; it has been found equal to $0.2 \mathrm{~nm} / \mathrm{cm}$. In order to be sure that this value colild be used for the present study we have manufactured a laterally graded period LSM in the very same conditions that the laterally graded $\gamma \mathrm{LSM}$, whose characteristics are: 31 bilayers C/W, $\gamma=0.5$ (Fig. 5). The manufacturing technique has been described in reference [9].

Diffraction measurements performed at regularly spaced points of the sample made it possible to determine the period $d$ as a function of $x$. Figure 6 shows a plot $d$ versus $x$ for the first three order 


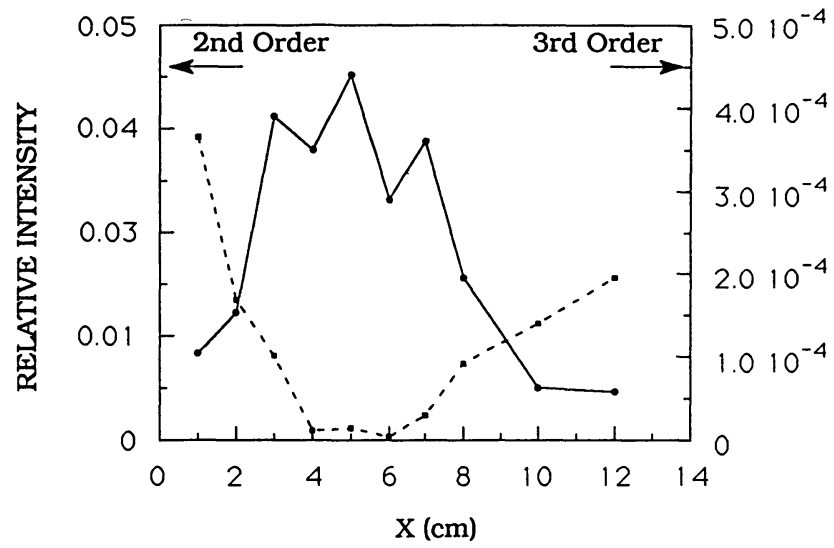

Fig. 4. - Relative intensities of the second order (- - - -) and the third order $(-\bullet)$ Bragg peaks with respect to the first one.

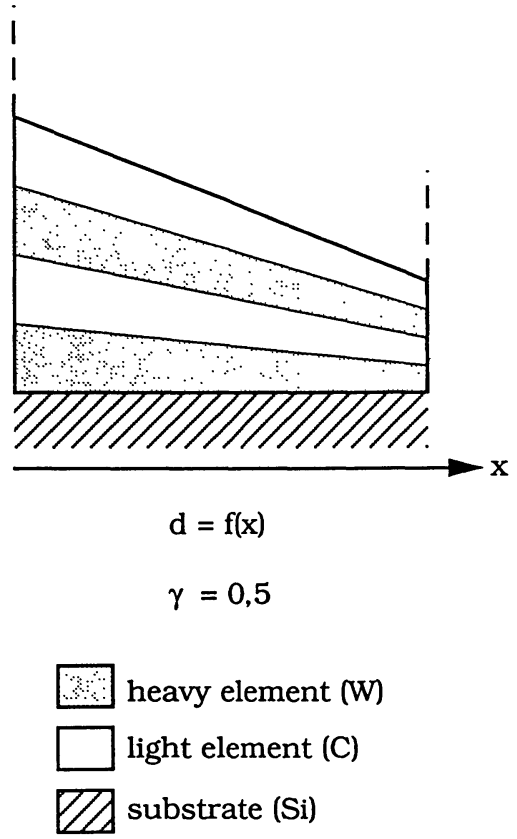

Fig. 5. - Theoretical profile for a laterally graded period LSM.

spectra. There is clearly a linear dependance between $d$ and $x$. The solid lines are least square fits of the data. The first order has larger deviations from the linear fit than the higher orders because we used the Bragg law which neglects the index correction. This correction is more significant in the first order. Because of the limits of the detector sensibility, the measurements for $x$ greater than $12 \mathrm{~cm}$ in the second order and $8 \mathrm{~cm}$ in the third order are very inaccurate.

In figure $6 \mathrm{~d}$, the calculation of $d$ have been carried out by means of the corrected Bragg law 


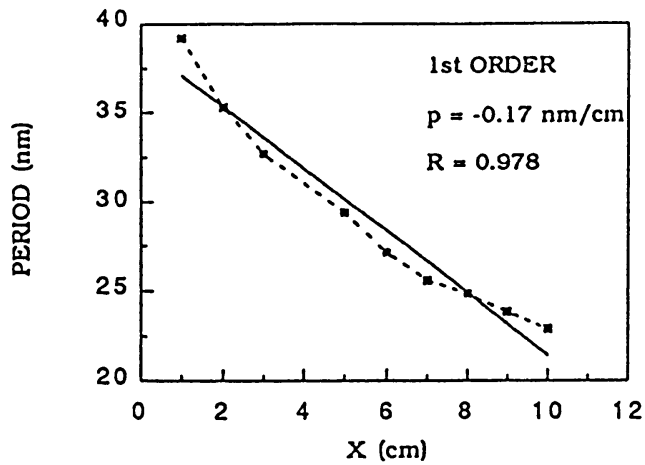

(a)

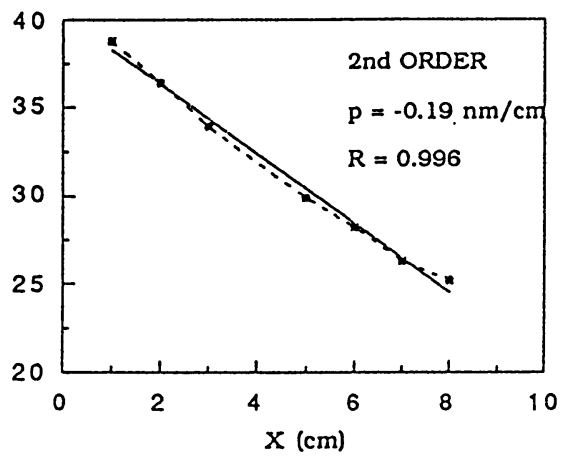

(b)

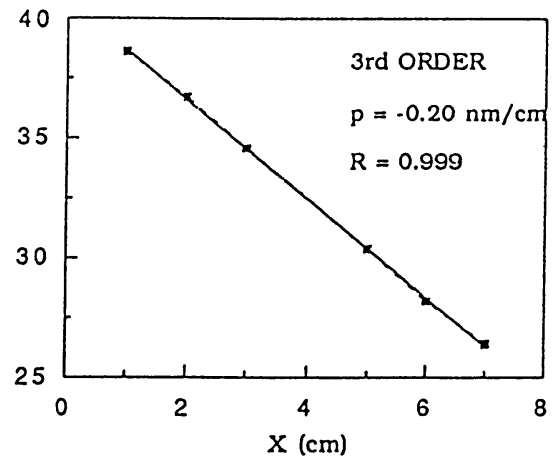

(c)

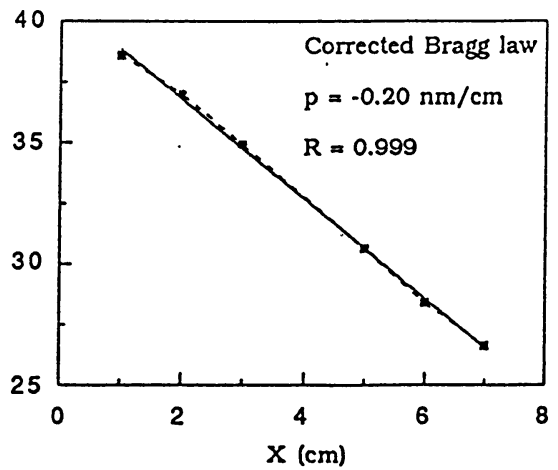

(d)

Fig. 6. - Diffraction measurements performed for a LSM with a laterally graded period for the first order (a), the second order (b) and the third order (c). For each order, we deduced the gradient $p$ and the correlation coefficient $R$. In (d), we used the corrected Bragg law and the first three orders for the calculations. - . - Experimental (from diffraction spectra); - Least square fit of the data.

like in paragraph 4 . In the range $0<x<8 \mathrm{~cm}$ the period $d$ decreases linearly up to $2.5 \mathrm{~nm}$ and the slope is found to be equal to $0.2 \mathrm{~nm} / \mathrm{cm}$, in good agreement with the expected value. Given that $\gamma=0.5$ we deduced that the slope of the interface between the $\mathrm{C}$ and $\mathrm{W}$ layers of the laterally graded $\gamma$ LSM is $0.1 \mathrm{~nm} / \mathrm{cm}$.

Thanks to the previous results and the estimate $\gamma=0.5$ for $x=5 \mathrm{~cm}$, the calibration curve relating $\gamma$ to $x$ may be written

$$
\gamma(x)=0.5+(p / 2 d)(x-5)
$$

with $x$ in $\mathrm{cm}$.

Given that $d=3 \mathrm{~nm}$ and $p=0.2 \mathrm{~nm} / \mathrm{cm}$, it follows that:

$$
\gamma(x)=0.5+(x-5) / 30
$$

Hence, we have for instance $\gamma(0)=1 / 3$ and $\gamma(10)=2 / 3$. 


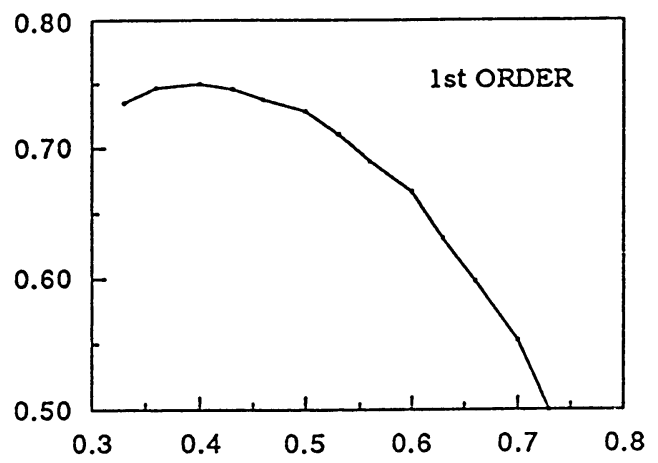

(a)
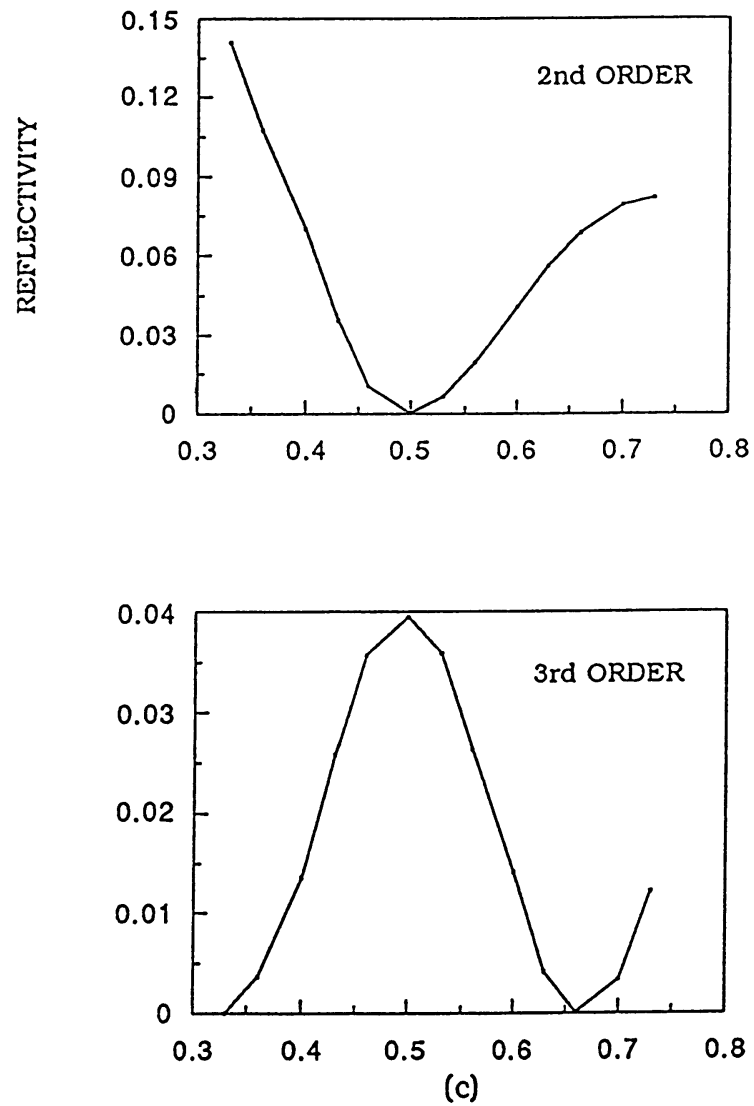

DIVISION PARAMETER $\gamma$

Fig. 7. - Computed reflectivity versus $\gamma$ for the first three orders. The first order is at a peak for $\gamma=0.4$ (a). The second order presents a relative minimum for $\gamma=1 / 2$ (b) and the third order for $\gamma=1 / 3$ and $2 / 3$ (c). 


\section{Computer simulations.}

The experimental measurements can be compared with modeling calculations performed using a recursive procedure initially developped by Paratt $[18,19]$.

Each point $x$ of the sample is equivalent to a sample with a period $d=3 \mathrm{~nm}$ and a division parameter $\gamma(x)$. Our experimental points belong to the range $0<x<12 \mathrm{~cm}$ with steps of $1 \mathrm{~cm}$. According to our experimental law of $\gamma$ versus $x$, we have considered for the division parameter the range $1 / 3<\gamma<11 / 15$ with steps of $1 / 30$.

Thus, for each value $\gamma(x)$, we have simulated a sample with the following parameters:

$$
\begin{aligned}
& d_{\mathrm{H}}=d_{\mathrm{W}}=3 \gamma(x) \mathrm{nm} \\
& d_{\mathrm{L}}=d_{\mathrm{C}}=3(1-\gamma(x)) \mathrm{nm}
\end{aligned}
$$

and we have computed the reflectivity for the three first order as a function of $x$ (Fig. 7).

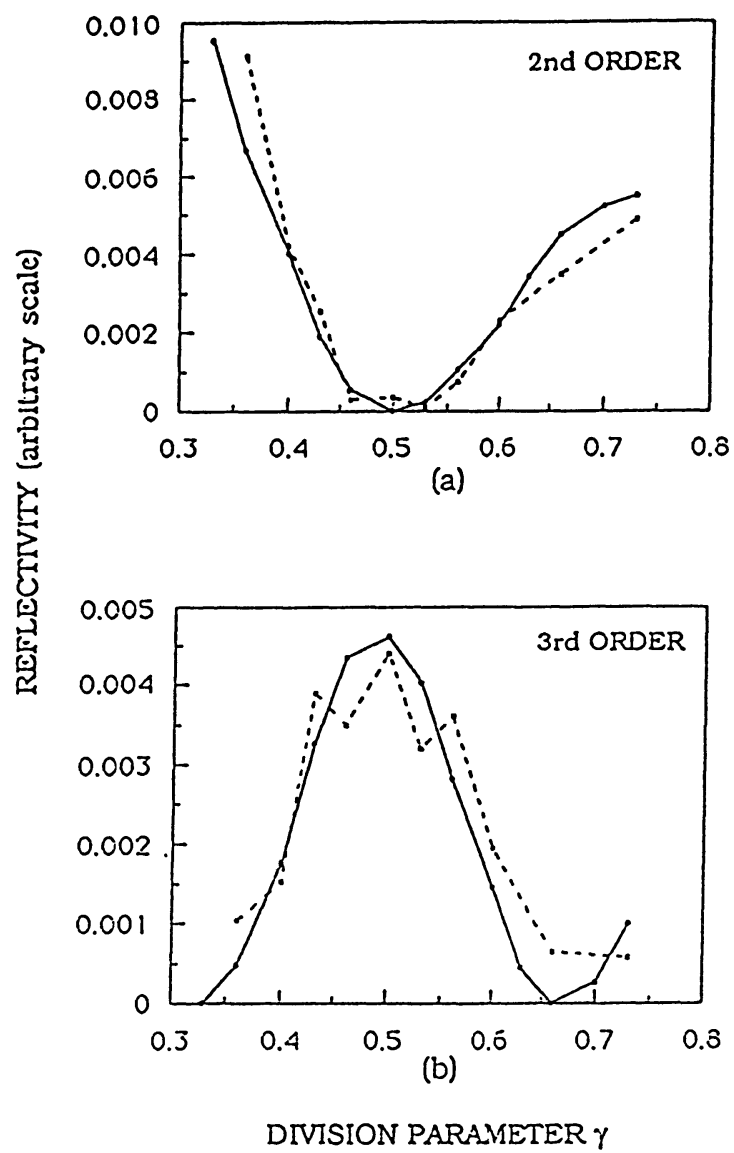

Fig. 8. - Reflectivity versus $\gamma$ for the second order (a) and the third order (b). - - - - Experimental (X-ray diffraction); - - Computer simulation.

It may be seen that the first order is at a peak for $\gamma=0.4$. The second order presents a relative minimum for $\gamma=1 / 2$ and the third order for $\gamma=1 / 3$ and $\gamma=2 / 3$. These results are in agreement 
with the DT predictions.

Finally, figure 8 shows the experimental and the computed curves for each order on the same scales to emphasize their correlations.

For the second order, it follows from the curve that the correlation is satisfying even if there is a relative disagreement for the last points $(x=10 \mathrm{~cm}$ and $x=12 \mathrm{~cm})$ where the thickness of the metal is more important. In this region, the absorption increases and consequently the reflectivity decreases.

We already noted that the experimental curve was very noisy for the third order. But with regard to the very low reflectivities in this order, the trend of this curve is correct and accounts for the phenomena predicted by the theory.

\section{Transmission electron microscopy study (TEM).}

In order to confirm the results presented in the previous sections, we undertook a transmission electron microscopy (TEM) analysis. Indeed, TEM analysis yields a direct solution by image formation from electron scattering. It provides local information on layer thicknesses, local periodicity, roughness of layers...

Investigation of multilayer structures in cross section by means of a TEM is difficult because of their thickness configuration. Some years ago, it has been shown that it was possible to observe a multilayer in transmission electron microscopy $[16,20]$. The procedure is as follows. After preparing a multilayer, a microcleavage technique makes it possible to cleave thin wedges from the multilayer. These wedges are deposited onto microscope grids and are orientated relative to the incident beam in such a way that their cross sections become observable. If the wedges are thin enough it is possible to obtain a good picture of the cross sections [21].

In this paper we used another procedure to prepare cross-sectional TEM specimens [22]. The samples were first glued together face to face, and then a mechanical thinning to $\approx 50 \mu \mathrm{m}$ was undertaken. Finally, argon-ion milling at liquid-nitrogen temperature was used to thin the specimens to electron transparency until a thickness $<100 \mathrm{~nm}$.

The TEM observations were performed with the electron beam parallel to the Si (111) axis using a Philips EM 400 microscope and operated at $100 \mathrm{keV}$ electron beam energy.

Three samples are presented here. For each of them, we obtained bright-field pictures and diffraction patterns. These regions are equivalent respectively with estimated values of the $\gamma$ parameter of $1 / 3$ (sample A), 1/2 (sample B) and 2/3 (sample C) (Fig. 9).

We recall that the multilayers were deposited onto (111) silicon substrates (silicon single-crystal wafer $15 \mathrm{~cm}$ in diameter). For each sample, we used an optical comparator to measure the thicknesses of tungsten and carbon respectively. We deduced the mean period which must stay approximately constant for the three specimens and the mean parameters $\gamma$ (Tab. II).

The mean parameters $\gamma$ deduced from the electron micrographs are in good agreement with those estimated from X-ray measurements. These results are quite conclusive given that in the case of electron microscopy, we investigated a very small region of the sample compared with the area scanned by the X-ray beam during a characterization by the diffractometer.

There is another way to measure the LSM's mean period with improved accuracy. Indeed, because the LSM's profile exhibit a regular periodicity, it is easy to obtain grating diffraction patterns by means of an electron beam nearly parallel to the multilayer planes.

For the calculation, the microscope has to be calibrated. Actually, we use the fact that LSMs are deposited onto well-oriented silicon (111) substrates. This allows us to obtain the LSM's diffraction and the substrate diffraction on the same pattern, the latter diffraction acting as a 
(A)

(B)

(C)

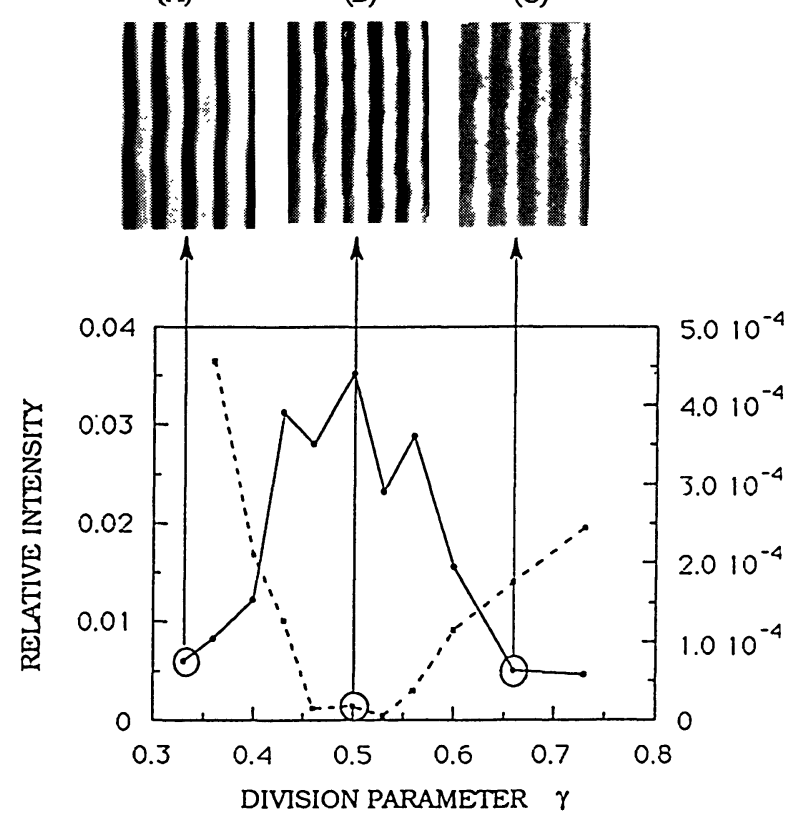

Fig. 9. - Photomicrographs of the cross section for three samples from the laterally graded $\gamma$ LSM. The division parameters are respectively $\gamma=1 / 3$ (sample A), $\gamma=1 / 2$ (sample B) and $\gamma=2 / 3$ (sample C).

Table II. - Characteristics (period and division parameter) of the laterally graded $\gamma$ LSM deduced by the three methods: $X$-ray diffraction, TEM and electron diffraction.

\begin{tabular}{cccccccc}
\hline & & \multicolumn{2}{c}{ Mean $\gamma$} & \multicolumn{3}{c}{ Mean Period (nm) } \\
Sample & $\mathrm{x}(\mathrm{cm})$ & $\begin{array}{c}\text { X ray } \\
\text { Diffraction }\end{array}$ & TEM & $\begin{array}{c}\text { X ray } \\
\text { Diffraction }\end{array}$ & TEM & $\begin{array}{c}\text { Electron } \\
\text { Diffraction }\end{array}$ \\
\hline A & 0 & 0.33 & 0.36 & 3.3 & 3.5 & 3.28 \\
B & 5 & 0.50 & 0.49 & 3.1 & 3.5 & 3.35 \\
C & 10 & 0.66 & 0.63 & 2.9 & 3.9 & 3.62 \\
\hline
\end{tabular}

reference. It's then possible to deduce the LSM's mean period $d$ by using the equation:

$$
D d=d_{111} D_{111}
$$

where $D_{111}$ is the (111) silicon diffraction-spot spacing and $d_{111}$ is equal to $0.3138 \mathrm{~nm}$. Figure 10 shows a complete multilayer stack from our sample with its diffraction pattern. We deduced the mean period from measurements on each side of the zeroth diffraction order and for the first three diffraction orders: $d=3.8 \mathrm{~nm}$.

In table II, we report the mean period calculated from the electron diffraction patterns for the three samples A, B and C. It must be emphasized that the values deduced from electron 


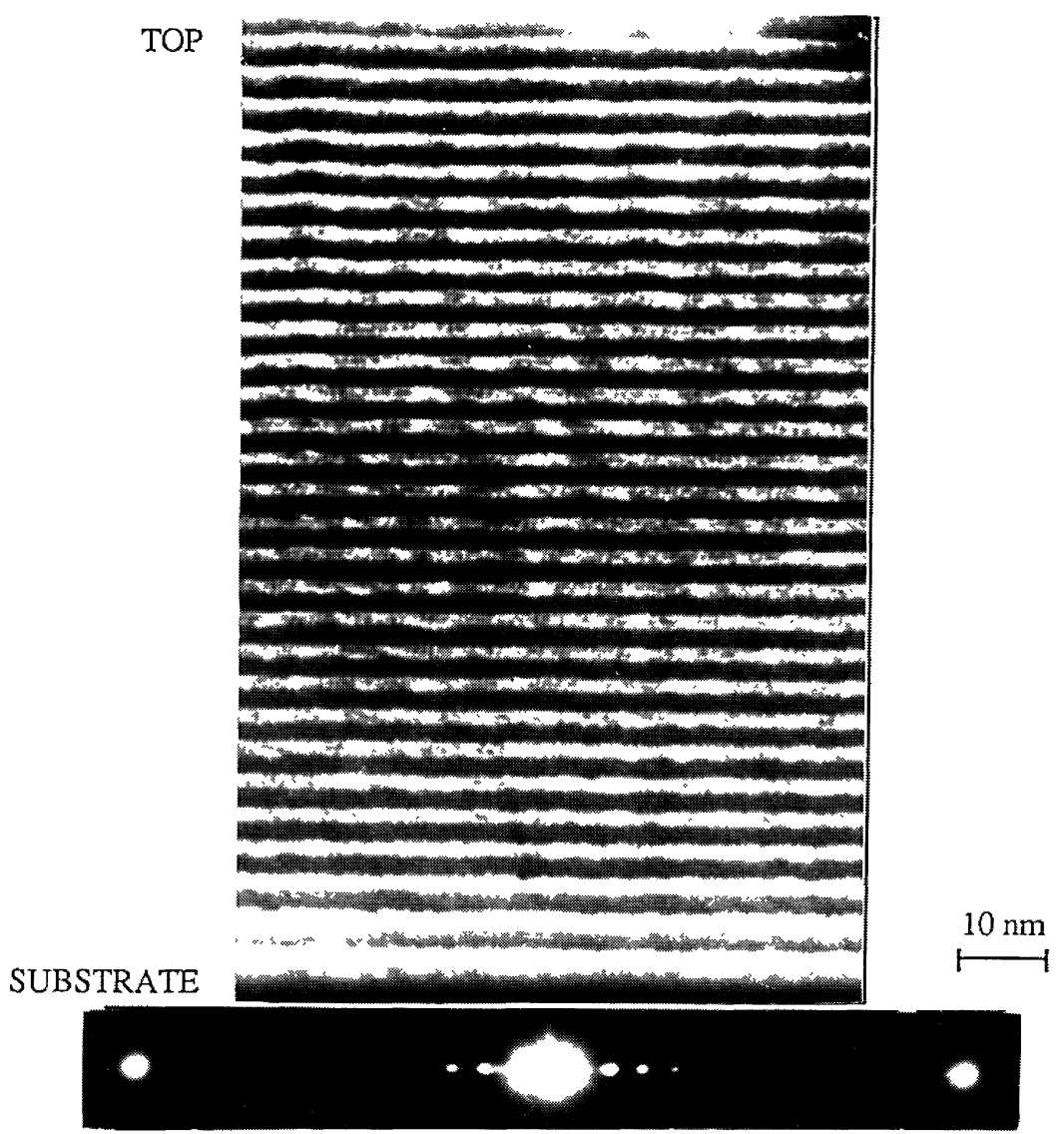

Fig. 10. - Photomicrograph of the cross section for a sample from the laterally graded $\gamma$ LSM which represents the complete multilayer stack with its electron diffraction pattern.

diffraction are much more accurate than those deduced from electron micrographs. They are in good agreement with the X-ray measurements.

\section{Conclusion.}

The results presented in this paper show that it is possible to manufacture laterally graded $\gamma$ LSM that really exhibit the properties predicted by the DT. Practical applications of such structures in $\mathrm{X}$-ray spectroscopy are obvious and this enhances the potentialities of the LSMs compared with the familiar crystals used in X-ray spectroscopy.

\section{Acknowledgements.}

We thank Dr B. Lazaridès for supplying the program for computer simulations. 


\section{References}

[1] Barbee T.W. Jr., "Multilayers for X-ray optical applications", in X-ray Microscopy, G. Schmahl and D. Rudolph, Eds. (Springer-Verlag, New-York, 1984) 144.

[2] Underwood J.H. and Attwood D.T., "The renaissance of X-ray optics", Phys. Today 37 (1984) 44-52.

[3] Spiller E. and Segmuller A., "The fabrication of multilayer X-ray mirrors", Ann. NY Acad. Sci. 342 (1980) $188-200$.

[4] Schuller I.K., "New class of layered materials", Phys. Rev. Lett. 44 (1980) 1597-1600.

[5] Barbee T.W. Jr., "Advances in multilayer X-ray/EUV optics: synthesis, performance and instrumentation”, Proc. SPIE 1159 (1989) 638-654.

[6] Barbee T.W. Jr. and Underwood J.H., "Solid Fabry-Perot etalons for X-rays”, Opt. Comm. 48 (1983) 161-166.

[7] Lepetre Y. Rivoira R., Philip R. and Rasigni G., "Fabry-Perot etalons for X-rays: construction and characterization”, Opt. Comm. 51 (1984) 127-130.

[8] Nagel D.J., Barbee T.W. Jr. and Gilfrich J.V., "Graded-layer thickness Bragg X-ray reflectors”, Proc. Soc. Photo-Opt. Instrum. Eng. 315 (1981) 110-117.

[9] Philip R., Rivoira R., Lepetre Y. and Rasigni G., "Laterally graded period layered synthetic microstructures”, Appl. Opt. 27 (1988) 1918-1919.

[10] Berrouane H., André J.M., Barchewitz R., Moreno T., Sammar A., Khan Malek C., Pardo B. and Rivoira R., "Experimental and theoretical performances of etched multilayer laminar grating in the $1 \mathrm{Kev}$ region", Nucl. Instr. Meth. A312 (1992) 521-530.

[11] Marshall G.F., "A unified geometrical insight for the design of toroïdal reflectors with multilayered optical coating: figured X-ray optics", SPIE 563 (1985) 114-134.

[12] Youn K.B., Sella C., Barchewitz R., Arbaoui M. and Alehyane N., "Absolute reflectivity measurements of multilayer interferential mirrors in the soft X-ray region", Opt. Comm. 77 (1990) 256-264.

[13] Zachariasen W.H., Theory of X-ray diffraction in crystals (Wiley, New-York, 1945).

[14] Underwood J.H. and Barbee T.W. Jr., "Layered synthetic microstructures as Bragg diffractors for Xrays and extreme ultraviolet: theory and predicted performance", Appl. Opt. 20 (1981) 3027-3034.

[15] Rosenbluth A.E., "Reflection properties of X-ray multilayer devices", PhD. Thesis, University of Rochester (New-York, 1982).

[16] Lepetre Y., Rasigni G., Rivoira R., Philip R. and Métois J.J., "Characterization of layered synthetic microstructures using transmission electron microscopy”, J. Opt. Soc. Am. 2 (1985) 1356-1362.

[17] Rosenbluth A.E. and Lee P., "Bragg condition in absorbing X-ray multilayers", Appl. Phys. Lett. 40 (1982) 466-468.

[18] Paratt L.G., "Surface studies of solids by total reflection of X-rays," Phys. Rev. 95 (1954) 359-369.

[19] Pelletier E., Klapisch M., Giacomo P., "Synthèse d'empilements de couches minces", Nouv. Rev. d'Opt. Appl. 2 (1971) 247-254.

[20] Lepetre Y. and Charaï A., "Multilayers observed by transmission electron microscopy", Thin Solid Films 105 (1983) 71-74.

[21] Lepetre Y., Schuller I.K., Rasigni G., Rivoira R., Philip R. and Dhez P., "Analytical electron microscopy of multilayered thin films using microcleavage”, Opt. Eng. 25 (1986) 948-953.

[22] Charaï A., Hörnström S.E., Thomas O., Fryer P.M., Harper J.M.E., "A comparison between aluminium and copper interactions with high temperature oxide and nitride diffusion barriers", J. Vac. Sci. Technol. A7 (1989) 784-789. 